\title{
公共图书馆文旅融合的实践及发展
}

\section{Practice and Development of Cultural and Tourism Integration in Public Libraries}

\author{
袁秀华 \\ Xiuhua Yuan
}

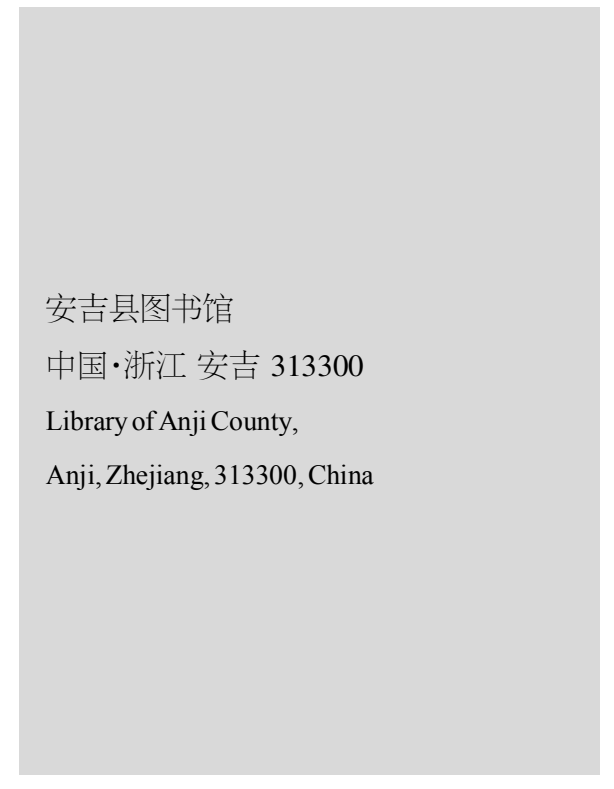

【摘要】在现代化社会的快速发展中, 公共图书馆越来越注重自身服务水平的提升。文 化和旅游的深度融合是新时期文化发展的必然趋势, 在很大程度上满足了人民群众的文 化需求。中国公共图书馆的文旅融合实践和发展推动着中国文旅融合发展事业的进步, 为 文旅融合发展提供了支持。文章主要对公共图书馆文旅融合的实践及发展进行了分析。

【Abstract】In the rapid development of modern society, public libraries are paying more and more attention to the improvement of their own service levels. The deep integration of culture and tourism is an inevitable trend of cultural development in the new period, which largely meets the cultural needs of the people. The practice and development of cultural and tourism integration in national public libraries promote the progress of the integrated development of Chinese cultural and tourism, and provide support for the integrated development of cultural and tourism. The article mainly analyzes the practice and development of cultural and tourism integration in public libraries.

【关键词】公共图书馆; 文旅融合; 实践; 发展

【Keywords \public library; integration of cultural and tourism; practice; development 【DOI】10.36012/lcs.v2i1.1477

\section{1 引言}

随着社会经济的快速发展, 人民群众的物质生活水平、知 识水平得到了很大提升，为了向人民群众提供优质的读书服 务,很多地区将公共图书馆和休闲旅游场所进行了融合, 为人 民群众的借阅提供了便利, 增加了休闲旅游场所的客流量 ${ }^{[1]}$ 。 基于此, 本文介绍了文旅融合对公共图书馆带来的影响, 分析 了公共图书馆发展现状，总结了公共图书馆文旅融合的实践 及发展策略。

\section{2 文旅融合对公共图书馆带来的影响}

\section{1 公共图书馆管理制度的变化}

在传统的公共图书馆服务过程中，图书馆管理方法和服 务方式无法满足现代化社会对图书馆的新要求。因此,公共图 书馆需要不断优化并改革管理制度和管理方式，进一步发展 和创新公共图书馆服务模式，更好地应对文旅时代带来的挑 战。在公共图书馆发展过程中, 相关管理部门需要严格落实法 人法理结构, 注重人事制度、分配制度和业务管理制度的优 化, 实行按岗按能付酬的方式, 以此为基础建立完善的激励制 度, 为公共图书馆工作人员提供平台, 营造积极进取的工作环 境, 建设高水平的图书馆队伍。

\section{2 图书馆信息平台的建立}

在互联网时代,信息呈现出碎片化、无序化的特点, 读者 利用互联网技术可以检索到更多的信息。公共图书馆需要利 用现代化信息整合技术、云计算技术和大数据技术,建立图书 馆信息平台,加大信息整合力度, 实现各项信息的融合, 为图 书馆信息管理工作、区域图书馆管理工作提供支持,形成图书 馆阅读、馆际互借、数字阅读等综合性图书馆信息服务平台, 为读者提供优质的信息服务, 更好地适应旅游高峰期读者人 数的增加。

\section{3 公共图书馆基础设施的完善}

为了更好地应对文旅融合带来的进馆游客激增的问题, 公共图书馆可以在条件和经费允许的情况下, 改造公共图书 馆的空间结构, 重新整合资源布局, 解决人流量增加的问题, 满足读者对文献的需求。同时, 公共图书馆需要完善图书馆内 部的指示性、指引性标志, 修订紧急疏散预案, 并做好预演和 演练, 及时更新硬件设施, 如办公设备、阅览座位和基础设施, 提高服务接待能力。

\section{4 图书馆工作人员管理与服务能力的提高}

图书馆工作人员的综合素质与公共图书馆管理和服务水 平之间的联系十分密切, 公共图书馆需要强化人才培养力度, 
制定完善的工作人员培养计划, 形成长效的工作制度, 将人才 培养作为常态工作。首先, 坚持以人为本的原则, 将图书馆工 作人员培养作为公共图书馆管理的重点工作; 其次, 定期开展 培训活动, 提高工作人员的政治思想觉悟和职业道德水平, 树 立读者第一、服务至上的工作理念, 根据读者的实际需求开展 管理和服务工作; 最后, 图书馆工作人员在日常生活中需要文 明、礼貌, 形成积极的工作态度, 加强对互联网技术的理解。

\section{5 为读者提供更多服务}

在互联网时代, 人工智能产品在不断普及, 人们获取信息 的方式发生了很大改变, 读者的阅读习惯和阅读兴趣也在发 生变化, 这就需要公共图书馆利用云计算技术、大数据技术开 展阅读、检索和跟踪活动, 明确读者的阅读习惯, 满足读者的 阅读需求 ${ }^{[2]}$ 。

\section{3 公共图书馆发展现状}

在新时期的发展中, 部分公共图书馆开设了公众号, 大力 宣传并推广公共图书馆开展的读书文化和读书活动, 吸引了 很多读者的关注。但是, 在公共图书馆的发展过程中, 部分工 作人员的服务意识相对薄弱, 这些工作人员往往只是整理书 籍, 很少为读者提供服务。另外, 部分公共图书馆未设置专门 为残障人士服务的部门, 导致这些人群在借书时遇到了一些 难题。部分公共图书馆设置了特殊人群阅览室, 但未能满足特 殊人群的实际需求。同时, 在工作过程中, 工作人员不能简单 地负责借还文献、整理文献, 还需要进行馆藏资源的数字化加 工, 为用户提供更多参考咨询服务。参考咨询服务主要是利用 数据挖掘、数据分析技术提炼相关信息, 将其转变成知识增值 性开发。为了实现这一目标, 图书馆管理部门需要加大智慧工 作人员的培养力度, 主要针对工作人员的专业技能、职业教 育,全面提升图书馆工作人员的综合素质。

\section{4 公共图书馆文旅融合的实践及发展对策}

\section{1 明确公共图书馆在文旅融合中的定位}

文旅融合指的是国家在机构改革战略下的既定方针, 公 共图书馆需要深入分析这一战略目标, 开展相关工作配合战 略目标的落实。在公共图书馆的发展过程中, 相关管理部门需 要积极探索文旅融合的优化途径, 树立“宜融则融, 能融尽融” 的理念, 深入分析图书馆所在地区的文化资源、旅游资源, 为 人民群众提供更加优质的文旅服务, 建设优质的文旅产品, 为 旅游产业的健康、持续发展提供支持。公共图书馆管理的重点 是实现文化的发展和传播, 具有公益性特点, 但在国家大力倡
导文旅融合的大背景下, 公共图书馆需要进行配合, 强化文化 和旅游业的融合, 推动旅游行业的进一步发展, 在旅游产业中 注人文化属性。

\section{2 打造地方文旅品牌}

在新时期的快速发展中, 各个地区的公共图书馆需要挖 掘并整理当地的历史文献、典籍, 将其和当地人民群众的文化 进行有效融合, 建设具备自身特色的地方文旅品牌; 还可以借 鉴先进的经验, 组织各个地区的公共图书馆开展交流、会议和 展览等, 并挖掘当地的文化传统和历史定位, 融入更多具备地 方特色的文旅产品, 如饮食文化、居住文化、建筑文化和民族 服饰文化等。例如, 浙江省安吉县开展了“旅游惠民周”活动, 在本次活动中, 很多亲子家庭到帐篷客酒店免费学习线装技 艺, 感受到了古代文化的魅力。通过开展“旅游惠民周”活动, 激发了很多孩子的兴趣, 具有很强的教育意义。另外, 孝文化 在中国源远流长, 是一种理念与精神, 是为人处事的立身之 本, 是社会责任的意识源头, 是中华传统文化的重要组成部 分, 而安吉县孝丰、孝源是二十四孝中“孟宗哭竹” “奉母得金” 两个故事的发源地。安吉县公共图书馆可以借此打造文旅品 牌, 吸引更多的游客, 为公共图书馆文旅融合的实践及发展提 供支持。

\section{3 构建公共图书馆研学旅游品牌}

研学旅游又被称为学习之旅, 其不仅是简单的学习旅程, 还将学习、考察、交流和旅游进行了有效融合, 是现代教育和 旅游发展下形成的一种新模式。在文化旅游一体化的快速发 展中, 研学旅游为文化旅游的发展和实现奠定了基础, 学生可 以参加学校暑期夏令营、社会志愿者活动等, 公共图书馆也可 以在旅游期间开展研学旅游活动。现阶段, 中国各个地区的公 共图书馆研学旅游活动的开展力度不断加强, 尤其是儿童图 书馆, 逐渐成为研学旅游的重要内容。

\section{5 结语}

综上所述, 为了应对现代化社会的发展, 公共图书馆需要 不断创新, 深入挖掘所在区域的人文资源和旅游资源, 建设文 化旅游品牌,为当地文旅融合提供优质服务。

\section{参考文献}

[1]王世伟.关于公共图书馆文旅深度融合的思考[J]. 图书馆,2019 (2):1-6.

[2]苟月华.公共图书馆文旅融合实践浅析[J].兰台内外,2019(23): $42-43$. 